\title{
Impact of age and comorbidity on risk stratification in idiopathic pulmonary arterial hypertension
}

\author{
Clara Hjalmarsson (10 ${ }^{1,2}$, Göran Rådegran ${ }^{3,4}$, David Kylhammar ${ }^{5}$, \\ Bengt Rundqvist ${ }^{1,2}$, Jonas Multing ${ }^{1,2}$, Magnus D. Nisell ${ }^{6}$ and Barbro Kjellström ${ }^{6}$ \\ on behalf of SveFPH and SPAHR
}

\begin{abstract}
Affiliations: ${ }^{1}$ Dept of Cardiology, Sahlgrenska University Hospital, Gothenburg, Sweden. ${ }^{2}$ Sahlgrenska Academy, Gothenburg University, Gothenburg, Sweden. ${ }^{3}$ Dept of Cardiology, Skåne University Hospital, Lund, Sweden. ${ }^{4}$ Dept of Clinical Sciences, Lund University, Lund, Sweden. ${ }^{5}$ Dept of Medicine and Health Sciences, Clinical Physiology, Linköping University, Linköping, Sweden. ${ }^{6}$ Cardiology Unit, Dept of Medicine, Karolinska Institute, Stockholm, Sweden.
\end{abstract}

Correspondence: Clara Hjalmarsson, Dept of Cardiology, Blå Stråket 3, 1st Floor, Sahlgrenska University Hospital, SE-405 30 Gothenburg, Sweden. E-mail: clara.hjalmarssondvgregion.se

@ERSpublications

Change in risk category at follow-up and specific comorbidity predict survival in IPAH across age groups http://ow.ly/EPQ530j765F

Cite this article as: Hjalmarsson C, Rådegran G, Kylhammar D, et al. Impact of age and comorbidity on risk stratification in idiopathic pulmonary arterial hypertension. Eur Respir J 2018; 51: 1702310 [https://doi. org/10.1183/13993003.02310-2017].

ABSTRACT Recent reports from worldwide pulmonary hypertension registries show a new demographic picture for patients with idiopathic pulmonary arterial hypertension (IPAH), with an increasing prevalence among the elderly.

We aimed to investigate the effects of age and comorbidity on risk stratification and outcome of patients with incident IPAH.

The study population $(\mathrm{n}=264)$ was categorised into four age groups: 18-45, 46-64, 65-74 and $\geqslant 75$ years. Individual risk profiles were determined according to a risk assessment instrument, based on the European Society of Cardiology and the European Respiratory Society guidelines. The change in risk group from baseline to follow-up (median 5 months) and survival were compared across age groups. In the two youngest age groups, a significant number of patients improved ( $18-45$ years, $Z=-4.613, p<0.001$; 46-64 years, $\mathrm{Z}=-2.125, \mathrm{p}=0.034$ ), but no significant improvement was found in the older patient groups. 5 -year survival was highest in patients aged $18-45$ years (88\%), while the survival rates were $63 \%, 56 \%$ and $36 \%$ for patients in the groups $46-64,65-74$ and $\geqslant 75$ years, respectively $(\mathrm{p}<0.001)$. Ischaemic heart disease and kidney dysfunction independently predicted survival.

These findings highlight the importance of age and specific comorbidities as prognostic markers of outcome in addition to established risk assessment algorithms. 


\section{Introduction}

Pulmonary arterial hypertension (PAH) is a deleterious, incurable disease that affects the small pulmonary arteries, with vasoconstriction and vascular proliferation leading to severe remodelling and an increased pulmonary vascular resistance. The increased right ventricular afterload results in right ventricular failure and ultimately death $[1,2]$.

The National Institutes of Health in the United States initiated the first large registry enrolling patients with idiopathic PAH (IPAH) 40 years ago [3]. This registry included 194 patients, with a mean age of 36 years, of whom $68 \%$ were women. Without effective treatments, the median survival time was estimated to be 2.8 years [1]. The first consensus guidelines for the diagnosis and treatment of IPAH were published in 1993 [4]. Now, new treatments are available that target the disease-related vasoactive pathways and influence symptoms, quality of life and survival $[5,6]$. However, contemporary reports from worldwide pulmonary hypertension $(\mathrm{PH})$ research registries show a new demographic picture for the IPAH population [7-9]. Prevalence among the elderly is increasing, with a mean age of 50-65 years reported at diagnosis [10-13]. The reason for this shift is not clear. Late-onset IPAH among older patients may include some degree of left ventricular diastolic dysfunction leading to a particular phenotype of "mixed" pre- and postcapillary PH [13]. These patients may share features of both IPAH and PH secondary to diastolic heart failure, as shown by OPITz et al. [14]. Improved evaluation of older patients with dyspnoea might also contribute to this demographic shift [15]. Interestingly, and in contrast to what is seen at many PAH clinics, most randomised controlled drug trials exclude elderly patients with multiple comorbidities $[16,17]$. Similarly, apart from the REVEAL score [18], which includes age $>60$ years and kidney dysfunction, the actual risk assessment instruments do not take into account age or comorbidity as prognostic markers. More data are required to support the use of risk equations and risk scores to assess subsequent risk [19].

Kylhammar et al. [20] recently validated the feasibility of the new European Society of Cardiology and the European Respiratory Society guidelines' instrument for risk assessment $[5,21]$ in a cohort of 530 patients with associated or familial/idiopathic PAH reported in the Swedish Pulmonary Arterial Hypertension Register (SPAHR). The findings showed that the recommended comprehensive risk assessment successfully discriminated patients' outcomes.

The aim of the present study was to further investigate the predictive value of the risk assessment instrument in the set-up of different age categories and associated comorbidities in a population with incident IPAH.

\section{Methods}

This study was based on data recorded in SPAHR [22]. All seven Swedish PAH centres report to SPAHR, enabling high national coverage [23]. SPAHR was initiated in 2008, after being approved by the National Board of Health and Welfare and the Swedish Data Protection Authority. All patients are informed about their participation and have the right to decline. Source data are regularly subjected to random monitoring onsite. The present study complies with the Declaration of Helsinki and was approved by the local ethics committee in Gothenburg, Sweden (Dnr. 2015/1002).

\section{Study population}

Patients with incident, adult IPAH registered in SPAHR between January 1, 2008 and June 29, 2016 were considered for the analyses. The day of diagnosis, confirmed by right heart catheterisation (RHC), was used as baseline. Follow-up was defined as the first registered visit at the PAH clinic occurring within 3-15 months after diagnosis.

The study population was categorised into four age groups: $18-45,46-64,65-74$ and $\geqslant 75$ years. The presence of seven common comorbidities was assessed: arterial hypertension, diabetes mellitus, ischaemic stroke, ischaemic heart disease, atrial fibrillation, obesity and kidney dysfunction.

\section{Variables}

The diagnosis of IPAH was determined by RHC, according to the 2009 [24] or 2015 [5, 21] guidelines criteria. The variables of interest extracted from SPAHR at baseline and follow-up comprised demographics, comorbidities, medical treatment, World Health Organization functional class (FC), data from RHC, 6-min walk distance (6MWD), blood biochemistry and echocardiography. Pulmonary capillary wedge pressure (PCWP) measurements by RHC, echocardiography and/or cardiac magnetic resonance imaging were performed to exclude $\mathrm{PH}$ due to left heart disease. Pulmonary function testing, including diffusing capacity of the lung for carbon monoxide (DLCO, \% of predicted value) and high-resolution computed tomography were performed to exclude $\mathrm{PH}$ due to lung disease. Chronic thromboembolic $\mathrm{PH}$ was excluded by pulmonary scintigraphy and/or pulmonary angiography. Creatinine levels were used to 
estimate glomerular filtration rate (eGFR) according to the Cockroft-Gault formula [25]. Kidney dysfunction was defined as an eGFR $<60 \mathrm{~mL} \cdot \mathrm{min}^{-1} \cdot 1.73 \mathrm{~m}^{-2}$ at baseline. Obesity was defined as a body mass index $>30 \mathrm{~kg} \cdot \mathrm{m}^{-2}$ at baseline.

\section{Risk assessment}

Risk assessment was based on the following specific variables according to the risk assessment instrument from the 2015 guidelines [5, 21]: FC, 6MWD, N-terminal pro-brain natriuretic peptide (NT-proBNP), right atrial area, mean right atrial pressure, pericardial effusion, cardiac index (CI) and mixed venous oxygen saturation $\left(\mathrm{S}_{\mathrm{vO}}\right)$. Each variable was graded from 1 to 3 where $1=$ low risk, $2=$ intermediate risk and $3=$ high risk. The sum of all grades was divided by the number of available variables for each patient, rendering a mean grade. The mean grade was rounded to the nearest integer, which was then used to define the patient's risk group. Details regarding this method for risk assessment have been previously published elsewhere [20].

\section{Statistical methods}

Fisher's exact test or Chi-squared test was used to compare categorical variables. For continuous data, between-group differences were compared using one-way ANOVA, with post hoc Bonferroni testing. All analyses were stratified by age group. Survival was analysed using Kaplan-Meier estimates and Cox proportional hazard regression, for which sex, comorbidity and change in risk category from baseline to follow-up were used as covariates. The results are presented as the hazard ratio (HR) with $95 \%$ confidence intervals. Change in risk group from baseline to follow-up in relation to age was compared using the Wilcoxon signed-rank test. The Z-score (also known as the standard score) was produced by using the deviation from the mean, in terms of standard deviation units, and was used for samples with $>20$ observations in order to obtain a standardised, normal distribution. Absolute values $>1.96$ were considered significant. p-values $<0.05$ were regarded as statistically significant (two-sided test). All statistical analyses were performed using SPSS Statistical Software Package, version 22.0 (SPSS Inc., Chicago, IL, USA).

\section{Results}

This analysis included 264 patients with IPAH. PAH-targeted therapy was initiated at baseline $(<3$ months from diagnosis) in 236 patients (88\%), of which 23 (10\%) fulfilled the criteria as responders to an acute vaso-reactivity test $[5,21]$ and were treated with a high dose of calcium-channel blocker. For the remaining 31 patients, treatment was recorded in SPAHR $>3$ months after diagnosis. Follow-up visits were available for 220 patients; of these, 87 patients had RHC. The median time from baseline to first follow-up was 5 months (interquartile range (IQR) 4-6 months).

\section{Baseline characteristics by age group}

Women were predominant in the two youngest age groups, and accounted for $\sim 50 \%$ or less of the patients in the oldest groups (table 1). The youngest patients, 18-45 years, were more often diagnosed with FC II and had the lowest body mass index, NT-proBNP level and systolic blood pressure, as well as the longest 6MWD and the highest CI, arterial saturation $\left(\mathrm{SaO}_{2}\right)$ and $\mathrm{SvO}_{2}$, compared to the other age groups. This group also had the highest eGFR and mean pulmonary arterial pressure (mPAP). In contrast, patients $\geqslant 75$ years had the lowest mPAP, the shortest 6MWD and the highest NT-proBNP level.

The DLCO significantly declined with age. Patients in the age group 18-45 years were more often treated with combination PAH-targeted therapy at baseline and less often used diuretics or oxygen treatment compared to the older age groups. Treatment was similar among the two oldest age groups.

\section{Risk assessment}

A median of seven variables (IQR 6-7) per patient were available for assessment at baseline and five (IQR 3-6) at follow-up. FC, 6MWT and at least one measure of right ventricular function (NT-proBNP, echocardiography and/or RHC) were available for $82 \%$ of patients at baseline and $84 \%$ of patients at follow-up. At baseline, $29 \%$ of patients $18-45$ years of age were in the low-risk group, compared to $22 \%$, $9 \%$ and $6 \%$ of the patients in the age groups $46-64$ years, $65-74$ years and $\geqslant 75$ years, respectively (table 1 ). In the two youngest age groups, a significant number of patients improved, moving from the intermediate or high-risk group at baseline to the low-risk group at follow-up (18-45 years $\mathrm{Z}=-4.613, \mathrm{p}<0.001$; 46-64 years $\mathrm{Z}=-2.125, \mathrm{p}=0.034)$. There was no significant difference in risk group distribution between baseline and follow-up in the two oldest age groups (65-74 years $Z=-0.707, p=0.480$; $\geqslant 75$ years $\mathrm{Z}=-0.832, \mathrm{p}=0.405$ ) (figure 1 ). 
TABLE 1 Baseline characteristics of the study population by age

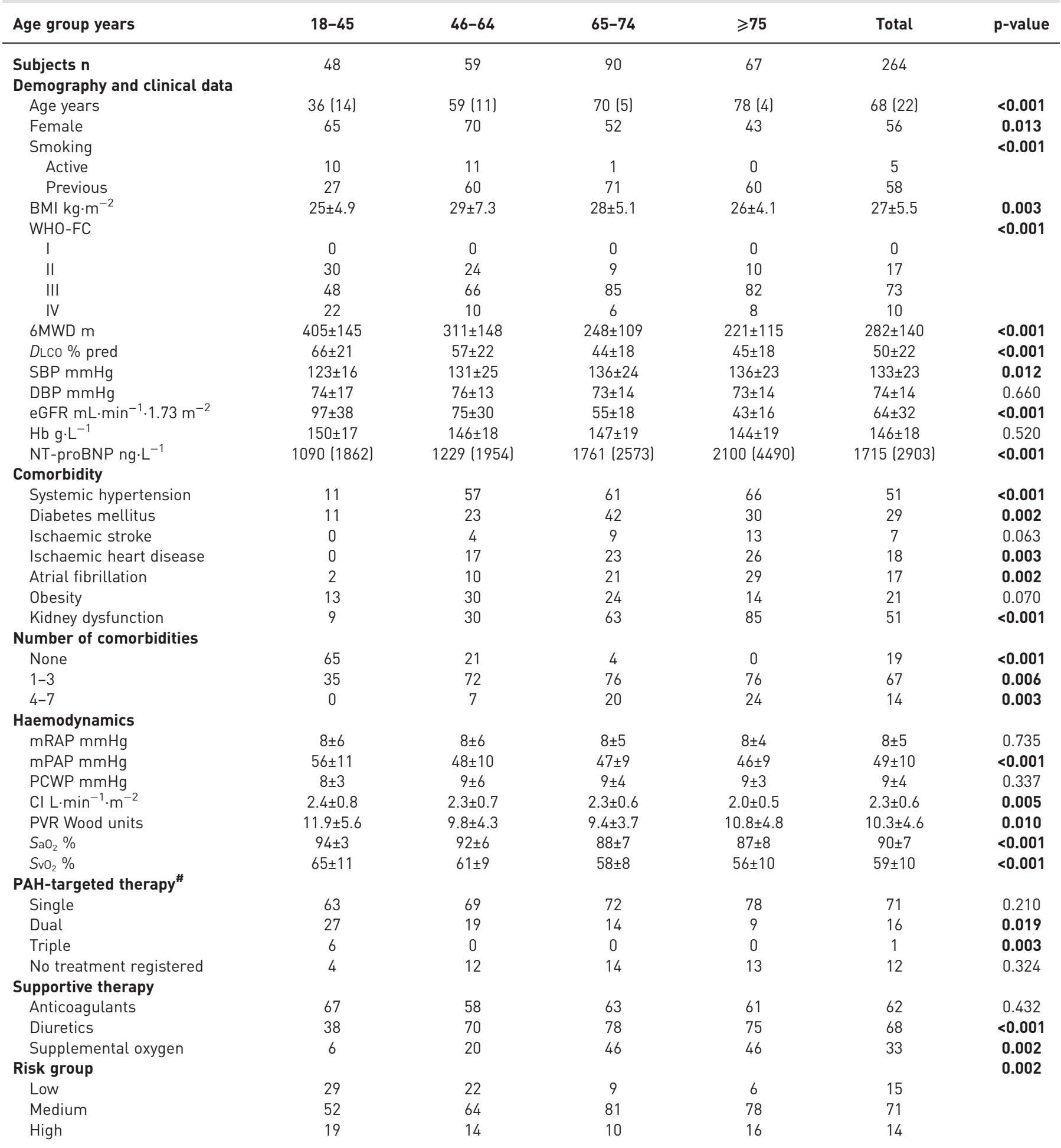

Data are presented as median (interquartile range), \% or mean \pm SD, unless otherwise indicated. BMI: body mass index; WHO-FC: World Health Organization functional class; 6MWD: 6-min walking distance; DLCo: diffusing capacity of the lung for carbon monoxide; SBP: systolic blood pressure; DBP: diastolic blood pressure; eGFR: estimated glomerular filtration rate; Hb: haemoglobin; NT-proBNP: N-terminal pro-brain natriuretic peptide; mRAP: mean right atrial pressure; mPAP: mean pulmonary arterial pressure; PCWP: pulmonary capillary wedge pressure; $\mathrm{Cl}$ : cardiac index; PVR: pulmonary vascular resistance; $\mathrm{SaO}_{2}$ : arterial oxygen saturation; $\mathrm{SvO}_{2}$ : mixed venous oxygen saturation; PAH: pulmonary arterial hypertension. ${ }^{\#}$ : started within 3 months from diagnosis. Bold indicates statistical significance at $p<0.05$. 

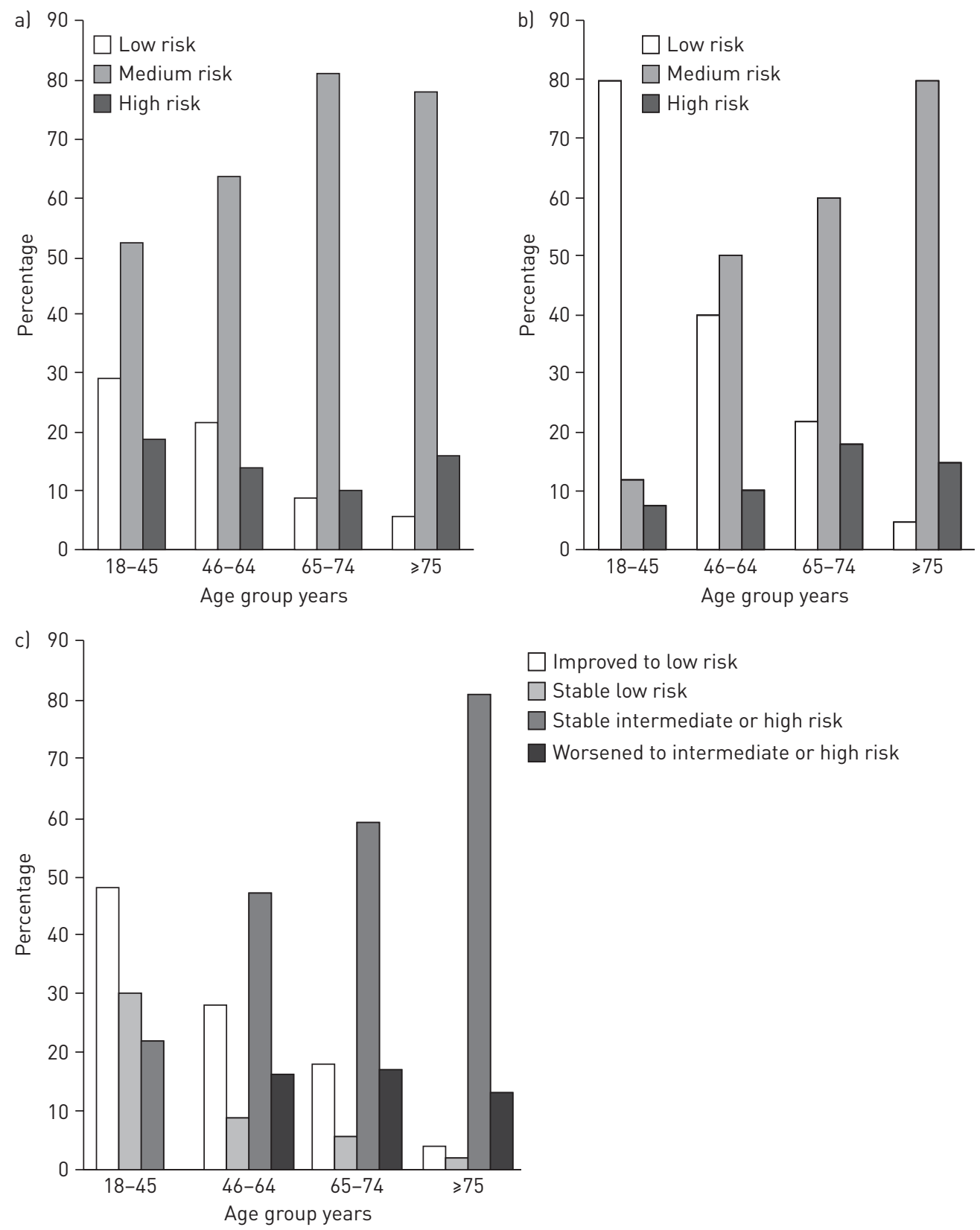

FIGURE 1 Risk assessment presented by age at a) baseline and b) follow-up, and c) change in risk group from baseline to follow-up.

\section{Comorbidity}

The comorbidity profile by age group is shown in table 1 . Comorbidity was more frequent in the two oldest age groups, in which $20 \%$ had at least four comorbidities. In a Cox proportional regression analysis, adjusted for sex and change in risk group from baseline to follow-up, ischaemic heart disease and kidney dysfunction were the only comorbidities that independently affected survival (table 2).

\section{Survival}

Up to study-end (median 73 months, IQR 37-133 months), 114 deaths and 11 lung transplants were recorded. Of these, 106 deaths and 10 lung transplants occurred within 5 years from baseline and 52\% of those who died or underwent transplant were women $(\mathrm{p}=0.217)$. The transplant-free 1-, 3- and 5-year survival rates for the whole study population were $87 \%, 67 \%$ and $58 \%$, respectively. Patients $18-45$ years had the highest transplant-free 5-year survival rate of $88 \%$, compared with $63 \%, 56 \%$ and $36 \%$ for patients in the groups $46-64,65-74$ and $\geqslant 75$ years, respectively $(\mathrm{p}<0.001)$. In an analysis adjusted for age 
TABLE 2 Cox proportional regression analysis adjusted for comorbidity, showing the relationship between mortality and explanatory variables

\begin{tabular}{llc} 
Explanatory variable & HR (95\% CI) & p-value \\
\hline Sex & $0.82(0.48-1.41)$ & 0.473 \\
Worsening of risk group from baseline & $1.75(1.14-2.69)$ & $\mathbf{0 . 0 1 1}$ \\
Hypertension & $0.89(0.51-1.56)$ & 0.685 \\
Diabetes mellitus type 2 & $1.01(0.56-1.82)$ & 0.973 \\
Atrial fibrillation & $1.00(0.48-2.10)$ & 1.000 \\
Ischaemic heart disease & $2.14(1.21-3.78)$ & $\mathbf{0 . 0 0 9}$ \\
Stroke & $2.00(0.85-4.74)$ & 0.114 \\
Obesity & $1.44(0.78-2.66)$ & 0.245 \\
Kidney dysfunction & $1.85(1.09-3.14)$ & $\mathbf{0 . 0 2 2}$
\end{tabular}

Worsening of risk group from baseline, ischaemic heart disease and kidney dysfunction were independent predictors of survival. Bold indicates statistical significance at $p<0.05$. HR: hazard ratio.

categories, survival was independently predicted by change in risk category from baseline to first follow-up visit (figure 2).

\section{Discussion}

The main findings of the present study illustrate that improvement in risk category at follow-up is a strong predictor of survival across all age groups. Young patients of 18-45 years had a high and significantly better 5-year survival rate than patients in the older age groups. In contrast, elderly patients were less often initially treated with combination PAH-targeted therapy and had a poorer outcome. They also exhibited a worse treatment response, which might be attributable to a different PAH phenotype, delayed diagnosis, less intense treatment or associated comorbidity. Ischaemic heart disease and kidney dysfunction were independently associated with poor prognosis.

Few studies have assessed the characteristics of patients with IPAH based on their age $[10,26]$ or the effect of age on treatment response [10, 27]. The COMPERA study used a cut-off age of 65 years [10]. The results were similar to the present study: younger patients were more often female and, despite a worse hemodynamic profile at baseline, had a lower FC, better NT-proBNP level and longer 6MWD. Another interesting observation was that $\mathrm{mPAP}$ at diagnosis declined with age, confirming the findings by HoEPER et al. [10]. A possible explanation for this would be that elderly people have worse adaptive mechanisms for increased right ventricle pressure load, and probably develop right ventricle failure at lower pulmonary

FIGURE 2 Cumulative survival of the study population adjusted for age and stratified by risk category.

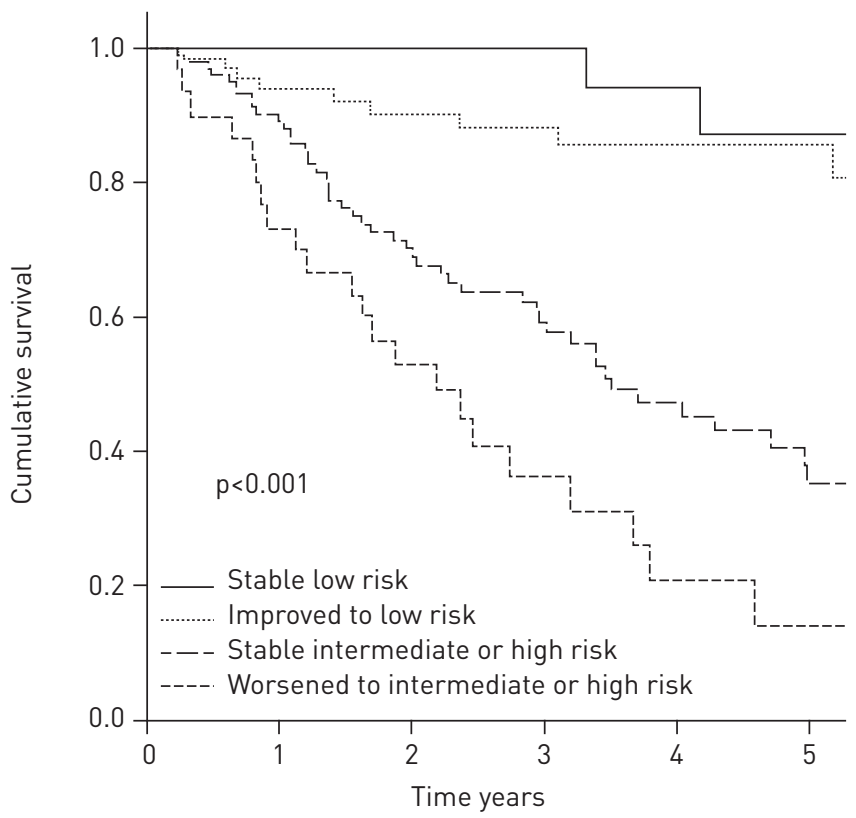


arterial pressures. This, in combination with comorbidity and frailty, may influence the patients to seek medical attention before the MPAP reaches higher levels.

Recently, an abbreviated version of the risk assessment strategy proposed by the current European $\mathrm{PH}$ guidelines was proven to accurately predict transplant-free survival in the French PAH registry population [28] and the COMPERA [29]. Additionally, work by Kylhammar et al. [20] showed that change in risk profile from baseline to follow-up is an even stronger predictor of survival in comparison to risk at baseline.

In the present study, patients aged 18-45 years had better outcomes than those in the intermediate age group (46-64 years), with a 5-year survival rate of almost $90 \%$. In a report from the PH registry of the United Kingdom and Ireland [26], the median age was 50 years and the estimated 5-year survival rate for patients $<50$ years was lower than in the present study; similarly, a study by D'Alonzo et al. [1] estimated a median survival time of 2.8 years. The discrepancy between the results of these studies and our study may be due to a different inclusion period (2000-2009 and 1985-1988, respectively, versus 2008-2016), with less-developed treatment strategies.

In 2016, data from six large randomised controlled trials including patients with IPAH and associated $\mathrm{PAH}$ were presented in a meta-analysis [27]. The results indicated that older patients were more often classified into New York Heart Association FC III-IV and had shorter 6MWD, but had better hemodynamic status at baseline than younger patients. These results, as well as those from the COMPERA study [10], are in accordance with our findings, suggesting that our patient population is highly representative of the IPAH population, according to the present definition.

The improvement in risk score from baseline to follow-up was greater for the younger than the older patients. Given that age and comorbidity are usually correlated, it is difficult to assess the exact contribution of each of these factors to outcome. However, while the number of associated comorbidities did not affect survival (data not shown), the type of comorbidity did: ischaemic heart disease and kidney dysfunction were strongly associated with poor prognosis. Among patients $\geqslant 75$ years, ischaemic heart disease and kidney dysfunction were, as expected, more frequent; apart from this, the demographic and comorbidity profiles in this group were very similar to those of the 65-74 years group. Thus, the clinical improvement, estimated as change in risk category from baseline to follow-up, was smaller and survival worse in the oldest group. To some extent, this might reflect the natural effect of age, as well as the deleterious effect of specific comorbidities on outcome, but it could also be due to delayed diagnosis, or to a different "PAH phenotype" with worse treatment response. It is very important to take into account the difference in treatment strategies, because older patients were less often treated with initial combination PAH-targeted therapy at baseline, probably due to biased allocation of treatment or to worse tolerability among older patients.

While it is well known that comorbid conditions can affect the course of many underlying disease states, data relating comorbidity to outcome in IPAH patients are scarce [10, 12, 30]. TRIP et al. [31] reported that a severely reduced DLCO in IPAH is associated with advanced age and greater tobacco exposure and it relates to worse exercise performance and decreased survival. A study from the REVEAL registry found that of seven common comorbidities, only diabetes and obstructive pulmonary disease were associated with an increased risk for death [12]. However, other diseases common among the elderly, e.g. atrial fibrillation, ischaemic heart disease, stroke and reduced kidney function, were not included in this analysis. This might be explained by the mean age being $<50$ years in the REVEAL registry study [7].

Serum creatinine has previously been associated with a worse hemodynamic profile and has been suggested as an independent predictor of mortality in PAH patients [32]. However, serum creatinine is a rough measure of kidney function, and can be influenced by other biological factors. Moreover, few studies have investigated the impact of renal function on outcome in IPAH patients $[33,34]$, and those studies included heterogeneous populations, with $\mathrm{PH}$ of mixed aetiologies. In the present work, ischaemic heart disease and kidney dysfunction $\left(\mathrm{eGFR}<60 \mathrm{~mL}^{-1} \cdot \mathrm{min}^{-1} \cdot 1.73 \mathrm{~m}^{-2}\right.$ ) were the only independent predictors of survival among the investigated comorbidities, suggesting that these two important comorbidities should be taken into account when making prognostic evaluations.

\section{Strengths and limitations}

One of the strengths of the present study is that it includes only patients with incident IPAH from 2008 forwards, thus giving an insight into an aetiologically homogenous group at a time with modern treatment strategies. All PAH centres in Sweden participate in SPAHR, allowing for a national coverage of $89 \%$ (for 2015). Registry data reflect patients seen in clinical practice and offer a real-life perspective as compared to data collected in randomised clinical trials. Categorising the study population in four age groups allows a more accurate description of comorbidity profile and outcome than in previous investigations. 
The limitations of the present work are those typically associated with observational registry studies, e.g. selection bias, lack of standardisation of registered variables and missing follow-up data. Another limitation is the relatively small size of the study population; however, in light of IPAH being an unusual disease, it is difficult to achieve large national study groups.

\section{Conclusion}

Improvement in risk category at follow-up was a strong predictor of survival across the age groups. The survival rate among young IPAH patients in the present study was considerably higher than previously shown, likely reflecting the improvement in modern treatment strategies. Elderly patients were more often treated with single rather than combination PAH-targeted therapy at baseline and had a poorer outcome. Ischaemic heart disease and kidney dysfunction independently predicted worse survival. The present study highlights the importance of age and specific comorbidity as prognostic markers of outcome, suggesting the usefulness of adding these parameters to previously established risk assessment algorithms.

Acknowledgements: We acknowledge the work of the SPAHR registrars at each of the seven PAH centres and Uppsala Clinical Research Centre, as well as Kjell Jansson at Linköping University Hospital, Björn Ekmehag and Björn Kornhall at Skåne University Hospital, Gerhard Wikström at Uppsala University Hospital and Stefan Söderberg at Norrlands University Hospital in Umeå for initiation and administration of SPAHR. We are greatly indebted to Max Petzold at Gothenburg University, for statistical advice.

Conflict of interest: C. Hjalmarsson reports personal lecture fees from Actelion Pharmaceuticals Sweden, outside the submitted work. G. Rådegran reports unrestricted research grants from Actelion Pharmaceuticals Sweden $\mathrm{AB}$ and GlaxoSmithKline, and personal lecture fees from Actelion Pharmaceuticals Sweden AB, Bayer Health Care, GlaxoSmithKline, NordicInfu Care and Sandoz/Novartis; G. Rådegran is, and has been, primary or co-investigator in clinical PAH trials for GlaxoSmithKline, Actelion Pharmaceuticals Sweden AB, Pfizer, Bayer Health Care and United Therapeutics, and has been involved in research advisory boards for Actelion Pharmaceuticals Sweden AB, Bayer Health Care, Eli Lilly and Sanofi-Aventis, outside the submitted work. D. Kylhammar reports unrestricted research grants from the Swedish Society of PH in cooperation with Actelion Pharmaceuticals Sweden AB, Pfizer and Bayer Health Care, and personal lecture fees from Actelion Pharmaceuticals Sweden AB and GlaxoSmithKline, outside the submitted work. B. Rundqvist has been primary or co-investigator in clinical PAH trials for GlaxoSmithKline, Actelion Pharmaceuticals Sweden AB, Bayer Health Care and United Therapeutics, outside the submitted work. M.D. Nisell reports personal lecture fees from Actelion Pharmaceuticals Sweden AB, Pfizer, Bayer Health Care, NordicInfu Care, Novartis, AstraZeneca, GlaxoSmithKline, Boehringer Ingelheim and Takeda; M.D. Nisell has been the primary or co-investigator in clinical trials for Boehringer Ingelheim, AstraZeneca, Takeda, Novartis and United Therapeutics, and has been involved in research advisory boards for Actelion Pharmaceuticals Sweden AB, Bayer Health Care, NordicInfu Care and GlaxoSmithKline.

Support statement: During the initiation of SPAHR, Actelion Pharmaceuticals Sweden AB, Bayer Health Care, Eli Lilly Sweden, GlaxoSmithKline, NordicInfu Care and Pfizer gave financial support. Since 2011, SPAHR has qualified as a national quality register and the Swedish Association of Local Authorities and Regions gives financial support. The financial supporters had no role in the data collection, analysis or interpretation and had no right in disapproving the manuscript.

\section{References}

1 D'Alonzo GE, Barst RJ, Ayres SM, et al. Survival in patients with primary pulmonary hypertension. Results from a national prospective registry. Ann Intern Med 1991; 115: 343-349.

2 Swiston JR, Johnson SR, Granton JT. Factors that prognosticate mortality in idiopathic pulmonary arterial hypertension: a systematic review of the literature. Respir Med 2010; 104: 1588-1607.

3 Rich S, Dantzker DR, Ayres SM, et al. Primary pulmonary hypertension. A national prospective study. Ann Intern Med 1987; 107: 216-223.

$4 \quad$ Rubin LJ. Primary pulmonary hypertension. Chest 1993; 104: 236-250.

5 Galiè N, Humbert M, Vachiery JL, et al. 2015 ESC/ERS Guidelines for the diagnosis and treatment of pulmonary hypertension: The Joint Task Force for the Diagnosis and Treatment of Pulmonary Hypertension of the European Society of Cardiology (ESC) and the European Respiratory Society (ERS). Eur Heart J 2016; 37: 67-119.

6 McGoon MD, Benza RL, Escribano-Subias P, et al. Pulmonary arterial hypertension: epidemiology and registries. J Am Coll Cardiol 2013; 62: Suppl. 25, D51-D59.

7 Badesch DB, Raskob GE, Elliott CG, et al. Pulmonary arterial hypertension: baseline characteristics from the REVEAL Registry. Chest 2010; 137: 376-387.

8 Frost AE, Badesch DB, Barst RJ, et al. The changing picture of patients with pulmonary arterial hypertension in the United States: how REVEAL differs from historic and non-US contemporary registries. Chest 2011; 139: 128-137.

9 Shapiro BP, McGoon MD, Redfield MM. Unexplained pulmonary hypertension in elderly patients. Chest 2007; 131: $94-100$

10 Hoeper MM, Huscher D, Ghofrani HA, et al. Elderly patients diagnosed with idiopathic pulmonary arterial hypertension: results from the COMPERA registry. Int J Cardiol 2013; 168: 871-880.

11 Humbert M, Sitbon O, Chaouat A, et al. Pulmonary arterial hypertension in France: results from a national registry. Am J Respir Crit Care Med 2006; 173: 1023-1030.

12 Poms AD, Turner M, Farber HW, et al. Comorbid conditions and outcomes in patients with pulmonary arterial hypertension: a REVEAL registry analysis. Chest 2013; 144: 169-176.

13 Pugh ME, Sivarajan L, Wang L, et al. Causes of pulmonary hypertension in the elderly. Chest 2014; 146: 159-166. 
14 Opitz CF, Hoeper MM, Gibbs JS, et al. Pre-capillary, combined, and post-capillary pulmonary hypertension: a pathophysiological continuum. J Am Coll Cardiol 2016; 68: 368-378.

15 Shimony A, Fox BD, Afilalo J, et al. Pulmonary arterial hypertension in the elderly-clinical characteristics and long-term survival. Lung 2012; 190: 645-649.

16 Galiè N, Barbera JA, Frost AE, et al. Initial use of ambrisentan plus tadalafil in pulmonary arterial hypertension. N Engl J Med 2015; 373: 834-844.

17 Sitbon O, Channick R, Chin KM, et al. Selexipag for the treatment of pulmonary arterial hypertension. $N$ Engl J Med 2015; 373: 2522-2533.

18 Benza RL, Gomberg-Maitland M, Miller DP, et al. The REVEAL Registry risk score calculator in patients newly diagnosed with pulmonary arterial hypertension. Chest 2012; 141: 354-362.

19 Raina A, Humbert M. Risk assessment in pulmonary arterial hypertension. Eur Respir Rev 2016; 25: 390-398.

20 Kylhammar D, Kjellstrom B, Hjalmarsson C, et al. A comprehensive risk stratification at early follow-up determines prognosis in pulmonary arterial hypertension. Eur Heart $J$ 2017; in press [https://doi.org/10.1093/ eurheartj/ehx257].

21 Galiè N, Humbert M, Vachiery JL, et al. 2015 ESC/ERS Guidelines for the diagnosis and treatment of pulmonary hypertension: The Joint Task Force for the Diagnosis and Treatment of Pulmonary Hypertension of the European Society of Cardiology (ESC) and the European Respiratory Society (ERS). Eur Respir J 2015; 46: 903-975.

22 Swedish Pulmonary Arterial Hypertension Registry (SPAHR). Yearly report 2016. Uppsala, Swedish Association for Pulmonary Hypertension. www.ucr.uu.se/spahr

23 Radegran G, Kjellstrom B, Ekmehag B, et al. Characteristics and survival of adult Swedish PAH and CTEPH patients 2000-2014. Scand Cardiovasc J 2016; 50: 243-250.

24 Galiè N, Hoeper MM, Humbert M, et al. Guidelines for the diagnosis and treatment of pulmonary hypertension. Eur Respir J 2009; 34: 1219-1263.

25 Cockcroft DW, Gault MH. Prediction of creatinine clearance from serum creatinine. Nephron 1976; 16: 31-41.

26 Ling Y, Johnson MK, Kiely DG, et al. Changing demographics, epidemiology, and survival of incident pulmonary arterial hypertension: results from the pulmonary hypertension registry of the United Kingdom and Ireland. Am J Respir Crit Care Med 2012; 186: 790-796.

27 Rose JA, Cleveland JM, Rao Y, et al. Effect of age on phenotype and outcomes in pulmonary arterial hypertension trials. Chest 2016; 149: 1234-1244.

28 Boucly A, Weatherald J, Savale L, et al. Risk assessment, prognosis and guideline implementation in pulmonary arterial hypertension. Eur Respir J 2017; 50: 1700889.

29 Hoeper MM, Kramer T, Pan Z, et al. Mortality in pulmonary arterial hypertension: prediction by the 2015 European pulmonary hypertension guidelines risk stratification model. Eur Respir J 2017; 50: 1700740.

30 Frost AE, Farber HW, Barst RJ, et al. Demographics and outcomes of patients diagnosed with pulmonary hypertension with pulmonary capillary wedge pressures 16 to $18 \mathrm{~mm} \mathrm{Hg}$ : insights from the REVEAL Registry. Chest 2013; 143: 185-195.

31 Trip P, Nossent EJ, de Man FS, et al. Severely reduced diffusion capacity in idiopathic pulmonary arterial hypertension: patient characteristics and treatment responses. Eur Respir J 2013; 42: 1575-1585.

32 Shah SJ, Thenappan T, Rich S, et al. Association of serum creatinine with abnormal hemodynamics and mortality in pulmonary arterial hypertension. Circulation 2008; 117: 2475-2483.

33 Kaiser R, Seiler S, Held M, et al. Prognostic impact of renal function in precapillary pulmonary hypertension. J Intern Med 2014; 275: 116-126.

34 Navaneethan SD, Wehbe E, Heresi GA, et al. Presence and outcomes of kidney disease in patients with pulmonary hypertension. Clin J Am Soc Nephrol 2014; 9: 855-863. 